\title{
Les femmes dans le projet libéral de modernisation de l'Équateur (1895-1925)
}

Women in the liberal project of modernization in Ecuador (1895-1925)

\section{Emmanuelle Sinardet}

\section{(2) OpenEdition}

\section{Journals}

Édition électronique

URL : https://journals.openedition.org/clio/9925

DOI : $10.4000 /$ clio.9925

ISSN : 1777-5299

Éditeur

Belin

Édition imprimée

Date de publication : 31 décembre 2010

Pagination : 253-269

ISBN : 978-2-8107-0098-1

ISSN : $1252-7017$

Référence électronique

Emmanuelle Sinardet, « Les femmes dans le projet libéral de modernisation de l'Équateur

(1895-1925) », Clio. Femmes, Genre, Histoire [En ligne], 32 | 2010, mis en ligne le 31 décembre 2012, consulté le 30 avril 2022. URL : http://journals.openedition.org/clio/9925 ; DOI : https://doi.org/ $10.4000 /$ clio. 9925 


\section{Les femmes dans le projet libéral de modernisation de l'Équateur (1895-1925)}

Emmanuelle SINARDET

Lorsque le libéral Eloy Alfaro s'empare du pouvoir en 1895, il entend mener à bien un projet de modernisation des institutions et de transformation complète du pays sur les plans économiques, sociaux, politiques, religieux. Souveraineté populaire, séparation de l'Église et de l'État, implication économique de l'État, sont les éléments constitutifs du programme libéral en ce qui concerne le développement des institutions centrales. Mais pour obtenir le soutien nécessaire à ces réalisations, le programme doit aussi inclure un projet de cohésion sociale qui garantisse le consensus de la société civile. Les libéraux travaillent en ce sens en proposant une société égalitaire où s'effaceraient les disparités héritées de la période coloniale, grâce au respect absolu des droits naturels de l'homme, de l'autonomie individuelle et des libertés publiques. Ils promettent d'offrir à tous les possibilités d'une amélioration sociale. Â travers la création d'établissements publics de bienfaisance, l'État doit assumer des fonctions sociales et protéger le citoyen ${ }^{1}$. L'éducation, laïque, publique et gratuite pour le primaire, joue également un rôle central dans cet ambitieux projet. L'Équateur libéral ne diffère pas des autres pays d'Amérique latine, ni dans ses principes ni dans ses orientations : la modernisation du pays, objectif ultime, repose sur la consolidation du modèle de l'État-nation et la redéfinition de son rôle en faveur de responsabilités sociales accrues.

Toutefois, en Équateur, la révolution libérale et laïque représente une rupture idéologique bien plus marquée que dans les pays voisins,

1 Clark 1995. 
en raison de l'héritage conservateur encore prégnant du "garcianisme », tant dans le fonctionnement institutionnel que dans les mentalités ${ }^{2}$. Président de 1860 à 1875, García Moreno, catholique fervent, avait fait d'une Église mise au service de l'État l'outil d'une refonte institutionnelle. A contrario, le libéral radical Eloy Alfaro, influencé par le positivisme anglo-saxon et les théories de Spencer ${ }^{3}$, prétend rompre totalement avec cet héritage, non seulement par les mesures économiques et institutionnelles devant mettre l'Équateur sur la voie du "progrès » et de la «civilisation», mais aussi en œuvrant pour une "modernisation» des mentalités. Cette dernière repose notamment sur une révision du rôle des femmes au sein de la société. Le libéralisme équatorien lance ainsi une dynamique d'émancipation féminine et témoigne de l'apparition d'un "premier cycle du féminisme », selon l'expression d'Asunción Lavrin ${ }^{4}$. Certes, ce discours féministe surgit en Équateur plus tardivement qu'en Argentine, au Chili et en Uruguay où il se manifeste dès les années 1880, mais il illustre de la même manière l'émergence d'un sujet féminin défendant, comme dans les autres pays latino-américains ${ }^{5}$, son droit à s'exprimer publiquement et à participer aux affaires de la cité.

\section{Le projet libéral : les femmes actrices de la révolution}

Les femmes apparaissent de façon récurrente dans les discours du président charismatique Eloy Alfaro. Elles semblent occuper ainsi une place importante dans son projet de modernisation nationale, tant du point de vue économique que social. Cette nouvelle dimension du rôle des femmes fait même l'objet d'une réflexion spécifique de la part d'Alfaro, comme l'illustre son «Message sollicitant la protection spéciale de la femme et recommandant sa participation aux emplois publics » :

2 Sinardet 2006.

3 Les libéraux équatoriens ne se réclament pas d'Auguste Comte mais de l'utilitarisme de John Stuart Mill et surtout d'Hubert Spencer et de ce qu'on appellera plus tard le darwinisme social.

4 Lavrin 1995.

5 Morant 2006b. 
Rien n'est plus douloureux que la situation de la femme dans notre Patrie. Elle y est reléguée aux tâches domestiques; la sphère de son champ d'action intellectuel est très limitée et l'espace où elle peut gagner sa vie indépendamment et décemment l'est davantage encore. Lui ouvrir de nouveaux horizons, la faire participer aux bienfaits du travail... En un mot, élargir son champ d'action en lui offrant un avenir meilleur est un sujet que nous ne devons jamais laisser de côté.

[...] Il incombe à l'Assemblée de 1897 d'améliorer la protection de la femme en votant des lois qui émancipent l'Équatorienne de ce cercle si étroit dans lequel elle vit et lui donnent les moyens de s'élever à un niveau où elle pourra trouver en abondance toutes les ressources nécessaires à une subsistance honnêté ${ }^{6}$.

Soulignons que l'émancipation projetée se présente d'abord comme économique. Certes, il s'agit de remédier à la situation misérable des veuves ou des femmes abandonnées par leur compagnon, qui ne trouvent pas d'emploi leur permettant de vivre décemment. Mais, plus largement, les femmes doivent devenir productrices de la richesse nationale, hors de leur foyer. Dans son allocution, le président Alfaro insiste sur l'entrée massive des femmes sur le marché du travail, à commencer par les services publics : télécommunications, poste, douanes, éducation. Notons que le travail féminin évoqué ici est indissociable de l'apparition d'une première classe moyenne urbaine, soutien d'Alfaro. Comme dans les pays du Cône Sud, le modèle de développement agro-exportateur, parce qu'il implique une modernisation des structures administratives et économiques pour répondre au défi d'une meilleure intégration au marché mondial, suppose de repenser le travail féminin. À Guayaquil, port qui se consacre à l'exportation de la richesse du pays, le cacao, les femmes occupent déjà des postes dans l'administration portuaire et douanière et dans les différents commerces ${ }^{7}$. L'État centralisé, qui entend bien étendre à l'ensemble du territoire, en partie encore enclavé, les techniques modernes de communication, espère les voir investir plus largement la téléphonie et la télégraphie et contribuer ainsi à la réalisation du projet de développement national.

6 Novoa 1900 : t. IV.

7 Arboleda $1985: 9$. 
Les femmes de cette classe moyenne urbaine naissante sont aussi les premières à bénéficier des réformes éducatives lancées par l'État sécularisé et qui doivent les préparer à occuper les postes que les libéraux leur destinent. Les ambitions manifestées par le nouveau régime sont remarquables dans le contexte de l'époque. Il prétend généraliser l'accès à l'éducation primaire non seulement en l'étendant aux zones rurales et reculées, mais en l'ouvrant à de nouveaux publics, les Indiens et les femmes en premier lieu. Tous sont en effet perçus comme un potentiel inexploité, maintenu dans l'ignorance de façon inique. Si l'éducation des Indiens reste un vœu pieux durant la période libérale, celle des femmes, en revanche, se met en place. Car le rôle des femmes dans la modernisation de la société équatorienne n'est pas uniquement conçu comme économique, il a également une portée idéologique. Rappelons que la laïcité promue par le nouveau régime s'applique aussi à l'enseignement. Aussi, l'école laïque, publique, obligatoire et gratuite, doit remplir sa mission: saper l'influence de l'Église sur les esprits. En l'occurrence, il s'agit de détourner du projet conservateur les femmes, considérées comme plus enclines à se soumettre à l'autorité des prêtres.

Concrètement, les femmes doivent bénéficier des réformes à caractère pédagogique. Celles-ci prétendent renouveler totalement le contenu des programmes et les matières enseignées: les enseignements techniques et scientifiques d'une part, la pédagogie au service de la formation de l'esprit critique et de l'autonomie intellectuelle de l'élève d'autre part, sont les nouvelles priorités. Les enseignements des écoles de filles insistaient jusqu'alors sur une formation domestique, avec des matières comme la couture ou la broderie. Même si ces dernières sont maintenues dans les nouveaux programmes, tous les établissements, privés comme publics, ouvrent désormais aux filles les enseignements à caractère scientifique : il leur est reconnu les mêmes aptitudes que les garçons et le droit de les développer. En effet, l'ignorance féminine souvent dénoncée n'est pas considérée comme la conséquence d'une supposée infériorité intellectuelle mais comme le résultat d'un processus social. Dans son discours d'inauguration de l'École Normale pour filles, Pedro D. Pombar H. déclare en 1901 : 
La femme ayant les mêmes aptitudes que l'homme, pourquoi ne pas s'efforcer de les développer comme chez lui ? Büchner a dit : s'il s'avère un jour que les résultats du travail de la femme n'égalent pas ceux de l'homme, n'en rendez responsables ni sa fragilité naturelle ni son manque de capacité ; rejetez plutôt la faute sur l'éducation imparfaite qu'elle a reçue et sur l'oppression sociale qui pèse sur elle ${ }^{8}$.

L'intégration des filles au système scolaire progresse fortement. En 1928, 59215 des 128446 élèves du primaire sont des filles, soit $46 \%$, un taux qui traduit un changement spectaculaire dans le pays ${ }^{9}$. Le nouveau régime s'efforce en outre de créer des établissements d'enseignement secondaire féminins. Certes, à la fin des années 1920, les garçons restent largement majoritaires dans le secondaire. Mais ces créations sont en soi une avancée, car elles représentent une réelle innovation. Avant la révolution libérale, en effet, il existait bien des «collèges» de filles; toutefois la distinction entre «collèges» et " écoles» de filles ne signalait pas une différenciation de niveaux dans le cursus mais seulement une distinction sociale, les «collèges » de filles étant réservés aux classes privilégiées.

Dans le supérieur, les femmes sont désormais admises au Conservatoire National de Musique et aux Beaux-arts. Le régime crée également des écoles d'infirmières, d'obstétrique et de pharmacie pour les recevoir et les former. Surtout, les femmes font leur entrée à l'Université, non sans difficulté : un décret de juillet 1895 finit par autoriser Aurelia Palmieri, pionnière, à étudier à l'Université de Guayaquil, après que l'Université, divisée par sa demande, eut soumis le cas à l'Assemblée Nationale ${ }^{10}$. Si les réticences demeurent, l'arbitrage se fait en faveur des femmes comme l'illustre l'itinéraire de la première diplômée en médecine, Matilde Hidalgo. D’abord institutrice, elle souhaite s'inscrire à la Faculté de médecine de l'Université Centrale de Quito, ce que le recteur lui refuse, lui conseillant une spécialisation en pharmacie ou obstétrique, jugée plus conforme au rôle traditionnel féminin. Elle suit alors les trois premières années de son cursus de médecine à l'Université de Cuenca

\footnotetext{
$8 \quad$ Ministère de l'Éducation $1951: 51$.

9 Ministère de l'Instruction publique 1928 : cuadro final $\mathrm{n}^{\circ} 2$.

10 Estrada $1981: 48-49$.
} 
qui ne s'oppose pas à son inscription, jusqu'à ce que l'Université Centrale l'accepte et lui octroie, en 1921, le doctorat en médecine.

Appelées à jouer un rôle actif dans la modernisation de la société équatorienne, les femmes doivent devenir, au même titre que les hommes, les instruments de la réalisation des réformes, comme en témoigne la création des Écoles Normales laïques. En février 1901, deux Écoles Normales, innovations sans précédent en Équateur, sont inaugurées: une pour garçons et une pour filles, l'établissement Manuela Cañizares. Ce dernier bénéficie des mêmes moyens que l'établissement pour garçons: il possède ses propres bâtiments et offre des bourses aux élèves les moins fortunées. En outre, la formation offerte aux filles se base également sur les principes pédagogiques, alors nouveaux, des sciences de l'éducation. Selon ces dernières, enseigner est un métier qui nécessite des connaissances spécifiques, y compris pour les femmes. Il s'agit là d'une avancée réelle: les femmes ne deviennent pas enseignantes parce que de supposés instincts maternels les y prédisposeraient, mais reçoivent une formation spécialisée, la même que les hommes. Par rapport à la période précédente, leur présence dans les écoles n'est plus improvisée ; elle ne se limite plus non plus à la prise en charge des plus jeunes. Les femmes, professionnelles de l'enseignement, sont appelées à intervenir dans le secondaire également, non seulement comme enseignantes, mais aussi comme directrices partageant avec les hommes des responsabilités qui étaient jusqu'alors réservées à ces derniers.

Plus largement, ces nouvelles fonctions doivent servir la transformation des mentalités par le biais des normaliens qui sont appelés à remplacer le clergé dans l'éducation de la jeunesse. Les femmes, désormais, partagent avec les hommes la mission de diffuser les nouvelles valeurs portées par le libéralisme, en l'occurrence une morale laïque basée sur la tolérance et la raison. Les normaliennes sont perçues, au même titre que les normaliens, comme des soldats du progrès dans la lutte contre le fanatisme religieux :

Il vous incombe, sans aucun doute, de faire un bond décisif : rendez effective l'éducation laïque, réprimez les influences monastiques dans l'école et le collège, brisez les chaînes qui brident l'intelligence de la 
jeunesse équatorienne, en un mot émancipez l'Instruction Publique et vous aurez rendu le plus grand des services à la Patrie ${ }^{11}$.

Le nouveau rôle des femmes et les responsabilités idéologiques qu'elles doivent assumer posent sérieusement la question de la reconnaissance de leurs droits civils et civiques par l'État. Deux mesures constituent une première avancée. La première modifie la terminologie définissant le citoyen. La Constitution de 1883 excluait explicitement les femmes : seuls étaient présentés comme citoyens les hommes (varón) de nationalité équatorienne sachant lire et écrire, de plus de 21 ans, mariés ou veufs. La Constitution de 1897 supprime le terme varón, reconnaissant implicitement aux femmes le statut de citoyenne dans la mesure où elles remplissent les conditions requises. La seconde mesure s'inscrit dans la réforme laïque qui proclame le mariage civil en 1902. La loi autorise non seulement le divorce, mais permet à la femme de le demander.

Ainsi, le droit des femmes à participer au devenir national dans l'espace public est acquis en Équateur à l'avènement du $\mathrm{XX}^{\mathrm{e}}$ siècle. Toutefois, cette évolution concerne essentiellement une élite sociale et intellectuelle. L'ouverture de l'accès à l'éducation et aux emplois dans l'administration bénéficie aux femmes d'une classe moyenne naissante. La situation de la grande majorité des Équatoriennes reste inchangée, les mentalités évoluant lentement.

\section{Le débat sur les représentations de « la » femme}

Les lois de laïcisation ${ }^{12}$, qui suscitent l'opposition de l'Église et des conservateurs, donnent lieu à de vifs débats qui invitent l'ensemble de la société à s'interroger sur la famille et sa fonction sociale et, partant, sur celle de « la » femme. Les conservateurs ne sont pas opposés à des actions concrètes pour améliorer la situation des femmes et se montrent favorables à leur éducation, mais dans le cadre strict d'une

11 Ministère de l'Instruction publique 1900 : III.

12 Parmi les diverses mesures, citons la liberté de la presse en 1896 (jusqu'alors censurée, notamment par l'Église), la sécularisation des hôpitaux et des hospices en 1899, le mariage civil et le divorce en 1902, l'éducation laïque dans la Constitution de 1906, la nationalisation des biens de l'Église en 1908. 
féminité marianiste ${ }^{13}$. Convaincus d'une supériorité morale et spirituelle des femmes sur les hommes selon le modèle de la Vierge Marie, ils restent attachés à l'idée d'une supposée nature féminine censée définir le rôle de « la » femme comme pilier du foyer, cantonné dans la sphère familiale et privée. "La» femme perçue comme un être moralement supérieur, en raison de prédispositions naturelles pour la douceur, l'amour, l'indulgence, la compréhension, se voit confier une série d'obligations qui répondent à ces valeurs morales, comme l'éducation des enfants, la transmission des valeurs - notamment par son exemple vertueux - et la cohésion familiale ${ }^{14}$. Les conservateurs accordent ainsi aux femmes un rôle déterminant dans la construction nationale, comme garantes de la paix sociale et de la prospérité, mais uniquement au sein de la sphère privée ${ }^{15}$.

Les débats sur la loi instaurant le mariage civil en 1902, puis sa réforme en 1904, réactivent chez ses adversaires la représentation traditionnelle d'une supposée nature féminine douce, résignée et patiente. Parce qu'elle autorise aussi le divorce, la loi sur le mariage civil est pour les conservateurs une insulte faite à « la» femme : elle la priverait de sa mission première, veiller à la cohésion du foyer, et la dévoierait en la conduisant vers une vie jugée indécente. La loi est perçue comme un danger pour l'ordre public. Rappelons que le concubinage est alors très fréquent, surtout dans les milieux populaires, et que le mariage civil est utilisé essentiellement par des concubins pour légaliser leur union ${ }^{16}$. Dans la pratique, la nouvelle loi ne change rien. Ce qui scandalise l'Église, c'est la nouvelle représentation de «la» femme qu'elle promeut: celle-ci cesse d'être une figure morale idéalisée puisqu'il lui est reconnu la possibilité d'être faillible. Elle peut, de plus, cesser d'appartenir juridiquement à la sphère privée, comme fille, épouse ou mère, pour devenir, une fois

13 Handelsman 1978.

14 L'Équateur ne fait pas exception en Amérique latine, comme le montre pour le XIXe siècle Mary Louise Pratt : Pratt 1993.

15 Le rôle marianiste de la femme dans la construction nationale selon le projet conservateur est théorisé par Juan León Mera dans La Escuela Doméstica publié en 1880.

16 Moscoso 1996. 
divorcée, un individu autonome sans lien direct avec une figure de l'autorité masculine.

La sécularisation de l'éducation, en 1905 et 1906, ravive le débat. Federico González Suárez, évêque et leader de l'opposition conservatrice, s'efforce de dépeindre les ravages de l'éducation sans Dieu, condamnant en premier lieu la figure de la normalienne dans ses lettres pastorales. Cette dernière transgresse la représentation traditionnelle de «la» femme. Parce qu'elle relève d'institutions laïques, elle s'inscrit hors de l'univers moral qui prévaut pour définir la nature féminine. González Suárez insinue même qu'elle aurait abjuré la foi catholique pour devenir "athée » et « hérétique », avant de conclure sur la vision de «la»femme déchue, livrée au vice: «Quand la femme perd la foi, peut-elle conserver ne serait-ce que la pudeur ? Il ne reste rien à la femme qui perd la foi, [...] seulement... le dévergondage $»^{17}$.

Notons que cette peinture rejoint un autre topos de la représentation de «la » femme, comme un être naturellement enclin au vice. Cela n'est en rien contradictoire avec la représentation de «la» femme comme parangon de vertu. Car, seul un guide moral strict et fiable, en l'occurrence le prêtre, peut lui permettre de se sublimer pour devenir ce pilier du foyer, exemplaire et moralement supérieur. Dans cette perspective, «la» femme qui est en contact avec la laïcité est nécessairement perdue. À la figure de la Vierge Marie s'oppose alors celle d'Ève, tentée et tentatrice, qui introduit le désordre autour elle. González Suárez fait alors de la normalienne la figure de la contagion du vice aux enfants innocents. Cette Ève libérale entrainerait dans sa chute la famille chrétienne et la société entière.

Les représentations traditionnelles de « la » femme nourrissent ici un argumentaire censé s'opposer au processus de laïcisation en cours. Toutefois, le projet libéral n'est pas exempt d'ambiguités sur le rôle qu'il entend faire jouer aux femmes. En effet, les discours officiels et la presse libérale décrivent «la» femme comme un être supérieur pour ses qualités morales. Ainsi, outre les termes de «beau sexe», reviennent de façon récurrente les expressions «mère vertueuse »,

17 González Suárez 1927 : t. 1, 34-36. 
«abnégation », «épouse fidèle ». En 1901, les discours accompagnant l'ouverture de l'éducation aux femmes n'échappent pas à cette représentation :

Créée à l'image de l'ange pour de célestes destins, la femme, pour ce qui est du perfectionnement du genre humain, mérite bien qu'on lui offre tous les instruments nécessaires à sa grandiose mission $[\ldots]^{18}$.

La supériorité morale de «la » femme la destine à être, hors du foyer, le prolongement de ce qu'elle est censée être dans la famille. D'où les professions que les libéraux lui souhaitent voir embrasser : infirmière pour prendre soin des malades et des faibles avec dévouement ; obstétricienne soignant d'autres femmes dans le respect de leur pudeur ; institutrice au contact de l'enfance envers laquelle elle témoignera forcément de patience et de douceur. La conception d'une certaine délicatesse morale et physique détermine également les métiers que les femmes populaires peuvent occuper dans l'espace public : couturière, brodeuse, cuisinière ; en définitive, des métiers qui s'inscrivent dans le prolongement des activités domestiques féminines. De même, si le projet de modernisation libérale donne aux femmes les plus éduquées la possibilité d'occuper des fonctions dans les secteurs alors de pointe, comme la téléphonie et la télégraphie, c'est dans les bureaux et non sur le terrain. «La » femme qui travaille ne doit pas être une femme exposée au public : comme employée de bureau, caissière, comptable ${ }^{19}$ - des postes subalternes placés sous l'autorité d'une figure masculine. Lui sont réservés les lieux clos qui évitent, au nom du respect de sa pudeur et de sa fragilité physique, de parcourir les rues à la vue de tous. Le projet libéral, qui prétend émanciper «la» femme, porte en lui ses propres contradictions en limitant et orientant strictement les voies de l'émancipation.

Plus généralement, lorsque nous observons l'allocation des ressources financières, il s'avère que les efforts en faveur du développement de l'enseignement secondaire bénéficient d'abord aux garçons. Ce sont les nouveaux établissements publics de garçons qui absorbent la plupart des crédits. Les filles ont bien accès au primaire, mais leur entrée dans le secondaire, y compris pour les plus aisées, ne

\footnotetext{
18 Ministère de l'Éducation $1951: 51$.

19 Ministère de l'Instruction publique 1907 : VIII.
} 
va pas de soi, contrairement au Chili à la même époque. En 1925, l'éducation secondaire féminine est d'ailleurs essentiellement privée. Elle incombe encore, dans la pratique, aux institutions confessionnelles, l'Église apparaissant comme la garante d'une éducation morale toujours comprise comme indispensable aux filles. Le projet libéral pose lui-même les limites aux avancées qu'il prétend promouvoir : il ouvre des espaces aux femmes, mais uniquement dans la mesure où ces derniers ne contredisent pas les représentations traditionnelles d'un « éternel féminin ».

Les libéraux établissent ainsi une différence entre le «féminisme bien compris » et «le féminisme mal compris $»^{20}$ : ils défendent le premier, selon lequel l'entrée des femmes dans la vie publique ne saurait aller à l'encontre de leur rôle maternel. «La » femme n'existe pas indépendamment de sa famille. Certes, les avancées sont indéniables, mais dans le cadre contenu des assignations traditionnelles: maternité, décence, pudeur, sensibilité, fragilité, toujours pensées en relation avec la cellule familiale ${ }^{21}$. Les classes moyennes adhèrent à l'idéal d'une femme moderne et éduquée, dont le nouveau rôle passe par la connaissance et le savoir. Mais elles affrontent le défi de la modernité en le réconciliant avec la notion d'un honneur féminin préservé. La maternité reste à la fois l'essence de la féminité et la mission sociale première des femmes; elle conditionne la définition d'un «bon » féminisme.

En définitive, la principale différence entre les représentations libérales de «la»femme et les représentations conservatrices est l'abandon par les premières de toute référence religieuse. La morale laïque et positiviste que défendent les libéraux n'a de cesse de rappeler à « la » femme son devoir : comme procréatrice, il lui revient d'assumer les soins et l'éducation des enfants. «La» femme, en ce sens, reste la garante de la formation de bons citoyens, honnêtes et travailleurs, et la gardienne de la paix sociale. Elle constitue la figure qui donne le bon exemple et, par conséquent, reste soumise davantage que l'homme au contrôle éthique, au respect des normes admises. L'Équateur ne diffère pas des autres pays latino-américains à

\footnotetext{
20 Goetschel 2006.

21 Potthast \& Scarzanella 2001.
} 
l'époque libérale, étudiés par Isabel Morant ou Asunción Lavrin : la maternité définit le rôle des femmes et c'est au nom de la maternité que les femmes peuvent prétendre à une amélioration de leur statut ${ }^{22}$.

\section{L'émergence d'un sujet féminin}

Le discours sur le "féminisme bien compris », en dépit de ses limites, est adopté par les femmes pour développer un féminisme «maternaliste $~_{23}$ à partir duquel elles revendiquent un nouveau statut. Une parole féminine émerge, non seulement en littérature et en poésie mais dans la presse, avec la création de suppléments féminins et l'apparition de nouvelles revues : El Tesoro del Hogar (1890), La Mujer, revista mensual de Literatura y Variedades (1905), El Hogar cristiano (1906-1919), La Ondina del Guayas (1907-1910), La Mujer ecuatoriana (1918-1923), Flora (1917-1920), Brisas del Carchi (1919-1921). Ces revues féminines, encore peu nombreuses, sont réservées à des femmes lectrices et, partant, éduquées, les auteures étant elles-mêmes majoritairement issues de cette nouvelle classe moyenne urbaine. Elles témoignent néanmoins de l'apparition d'une pensée féministe en ce sens qu'elles rappellent que la subordination des femmes n'est pas naturelle mais déterminée par la société et que, par conséquent, des actions doivent être mises en œuvre pour améliorer leur condition. En raison de leurs divergences sur les stratégies à adopter, ces prises de parole ne sauraient représenter une pensée homogène ; néanmoins surgit un sujet féminin qui exige la participation des femmes dans l'espace public.

Les sujets abordés dans les revues sont essentiellement liés au foyer et à la maternité et rappellent l'importance de l'éducation féminine pour la modernisation de la société au sein de laquelle la femme-mère reste le pilier moral garant de l'éducation des enfants et de la transmission des valeurs. La démonstration peut s'avérer ambiguë : les auteures soulignent le rôle de premier plan que doivent jouer les femmes, mais autant en raison de leurs capacités maternelles qu'au nom de leurs aptitudes intellectuelles. Il reste impensable, y compris dans les milieux les plus progressistes, qu'elles puissent

\footnotetext{
22 Morant 2006a, Lavrin 1995.

23 Goetschel 2006 : 14-15.
} 
exister socialement sans maternité. Un consensus s'établit alors sur la nature du féminisme comme développement de qualités féminines envisagées dans leur complémentarité avec le masculin.

Toutefois, si la revue quiténienne Mujer insiste sur l'importance d'éduquer les filles en démontrant les apports bénéfiques pour le foyer d'une mère éduquée, elle n'a de cesse de rappeler que les indéniables qualités intellectuelles des femmes méritent d'être développées pour le bien de la femme également, pensée comme une individualité et non plus simplement comme le prolongement de sa famille. Apparus plus tardivement que dans les autres pays du Cône Sud, les premiers groupes féministes équatoriens, à l'instar du Centre Féministe Lumière du Pichincha en $1922^{24}$, font de l'éducation l'instrument indispensable à la libération du joug de l'ignorance. Émerge ainsi une revendication au droit à la réalisation personnelle dans des domaines autres que la maternité : les activités intellectuelles, artistiques et professionnelles. La remise en question du rôle traditionnel des femmes passe par la dénonciation de certains mécanismes sociaux: Mujer montre comment les femmes sont incitées à adopter et assumer les représentations de «la » femme fragile et délicate, en raison de l'éducation superficielle, de la pression sociale, de la reproduction des modèles traditionnels, des perspectives limitées qui leur sont offertes; si certaines paraissent frivoles, c'est parce que la société attend d'elles cette attitude.

Les limites du libéralisme se manifestent à l'évidence dans les difficultés de l'expression politique des femmes. Cette dernière, tardive, ne naît pas d'espaces libéraux mais des mouvements d'opposition au libéralisme, notamment socialistes et anarchistes. L'anarcho-syndicalisme, comme en Argentine, se développe. D’abord à Guayaquil, port commerçant ouvert aux influences favorables à l'égalité des sexes, où sont fondés des espaces féministes à l'instar des Centres La Aurora et Rosa Luxemburgo. En 1922, à l'occasion des grandes grèves organisées à Guayaquil par les syndicats de gauche naissants, les organisations féminines ouvrières acquièrent pour la première fois une visibilité politique ${ }^{25}$. Le jeune féminisme

\footnotetext{
24 Goetschel $2006: 45$.

25 Páez $1986: 49$.
} 
équatorien, loin d'être homogène, relève d'appartenances sociales et idéologiques diverses, mais il se montre divisé sur la question ouvrière ou la question paysanne. Son approche du travail est davantage liée aux problématiques de la classe moyenne. La Mujer ecuatoriana, organe du groupe «La Aurora», défend le travail féminin au nom de l'honneur féminin. Le travail garantit l'autonomie des femmes et leur évite la tentation d'une vie facile et légère ; rédempteur, il préserve leur dignité.

Quant à la revendication du droit de vote, elle se manifeste d'abord par une initiative individuelle. En 1924, la première femme médecin, Matilde Hidalgo, se présente devant les autorités publiques de la ville de Machala, où elle réside, pour s'inscrire sur les listes électorales. Elle profite de l'absence de précision du texte constitutionnel, déjà évoquée, quant au sexe des électeurs. Essuyant un refus, elle en appelle alors au Conseil cantonal, lequel en réfère auprès du ministre de l'Intérieur, qui donne finalement son accord. Toutefois, la Municipalité refuse de faire appliquer la décision et en appelle au Conseil d'État qui, de nouveau, donne raison à Matilde Hidalgo. Cette avancée pionnière s'explique par l'existence d'un climat favorable au vote des femmes parmi certains libéraux, qui y voient la possibilité d'élargir leur base politique - une tactique devenue nécessaire dans les années 1920 lorsque le régime est fragilisé et contesté. Matilde Hidalgo devient ainsi la première femme d'Amérique du Sud à exercer le droit de vote. Elle incarne cette femme moderne qui s'affirme en relation avec les réformes libérales : née en 1889, issue des classes moyennes, bénéficiaire de l'accès des femmes à l'éducation, elle investit en tant que médecin, par le soin, l'espace professionnel, conformément au « féminisme bien compris ». Les libéraux, au début du siècle, ont ainsi lancé une dynamique sur laquelle les femmes des années 1920 et 1930, filles de la révolution, s'appuient pour promouvoir elles-mêmes leur émancipation ${ }^{26}$ : en 1925 et 1926, candidate pour le parti libéral, Matilde Hidalgo est même élue conseillère municipale.

Bien qu'un précédent soit ainsi créé, les textes ne sont modifiés qu'en 1929. Ce sont finalement les gouvernements progressistes mis

26 Sinardet 2005. 
en place après le renversement du régime libéral en 1925 par la révolution julienne d'inspiration socialiste qui modifient la loi. Ils ont l'appui du parti conservateur qui espère capter une grande partie du vote féminin et alors qu'il n'existe toujours pas de féminisme suffragiste. Quoi qu'il en soit, les Équatoriennes, désormais au même titre que les hommes, obtiennent la pleine reconnaissance de leurs droits civiques.

Le regard porté par les libéraux sur «la» femme reste conservateur. Les considérations morales, aussi sécularisées soientelles, prévalent pour définir le comportement et le rôle social des femmes. Le féminisme "maternel», «bien compris », est opposé à celui «mal compris» qui voudrait faire assumer aux femmes les mêmes rôles que les hommes, notamment en politique. Si les libéraux prônent l'accès à l'éducation, la protection sociale et la reconnaissance de certains droits juridiques pour les femmes, ceux-ci restent pensés en harmonie avec le foyer et la maternité. Même si les femmes des classes moyennes commencent à investir le marché du travail et celles des milieux populaires sont de fait des chefs de famille subvenant aux besoins des enfants, les gouvernements libéraux insistent sur la fragilité de «la » femme et sur le devoir de «l'homme » de l'épauler. Leur méconnaissance de la vie quotidienne des Équatoriennes maintient «la » femme dans le vaste ensemble subalterne, que l'élite au pouvoir jusqu'en 1925 appelle «les masses", aux côtés des Indiens, des paysans métis pauvres, des premiers ouvriers urbains, des groupes considérés comme peu utiles à la nation si l'État n'intervient pas pour les guider, les éduquer et les protéger. Tous représentent des citoyens de seconde zone, incapables de jouer un rôle actif de façon autonome, sans la médiation du pouvoir libéral. Le discours émancipateur reste fondamentalement paternaliste à leur égard.

Pourtant, si la portée des réformes libérales reste limitée, elles marquent néanmoins une rupture en faisant pour la première fois de «la» femme une actrice du changement; surtout, elles permettent aux Équatoriennes de se penser en tant que telles, comme en témoigne leur participation croissante, après 1925, aux mouvements féministes continentaux desquels elles restaient jusqu'alors isolées. Un sujet féminin émerge qui entend jouer un rôle dans la construction 
nationale et finit par obtenir le droit de vote. Comme en Argentine et au Chili, les réformes libérales de modernisation nationale sont des clés pour comprendre l'affirmation du féminisme. En ce sens, le cas équatorien permet d'attirer le regard de l'historien sur le rôle de l'État dans la définition de nouveaux rôles et de nouvelles représentations.

\section{Bibliographie}

1897, Ley de Instrucción Pública, Quito, Imprenta del Gobierno.

1907, Ley orgánica de Instrucción Pública de Septiembre de 1906, Quito, Editorial Nacional.

1912, Ley orgánica de Instrucción Pública, Quito, Imprenta y Encuadernación Nacionales.

Clark Kim, 1995, «El bienestar social: experiencias del mercado interno en el Ecuador, 1910-1930 », Procesos, Revista Ecuatoriana de Historia, 7, p. 59-88.

CONTRERAS Jacqueline \& Rocío Rosero, 1988, Bibliografía sobre la mujer en el Ecuador, Quito, Instituto latinoamericano de investigaciones sociales.

Estrada Jenny, 1981, Una mujer total: Matilde Hidalgo de Prócel, Guayaquil, Universidad de Guayaquil.

Goetschel Ana María (comp.), 2006, Orígenes del feminismo en el Ecuador, Quito, Consejo Nacional de Mujeres (CONAMU)/FLACSO-Sede Ecuador/Comisión de género y equidad social del MDMQ/UNIFEM.

Gonzalez Suarez Federico, 1927, Obras Pastorales del Ilmo. Sr. Federico González Suárez, Quito, Imprenta del Clero, t. I-II.

HANDELSMAN Michael, 1978, Amazonas y artistas. Un estudio de la prosa de la mujer ecuatoriana, Guayaquil, CCE.

Jímenez De Vega Mercedes, 1981, La mujer ecuatoriana, Quito, Banco Central del Ecuador.

Lavrin Asunción, 1995, Women, Feminism and Social Change in Argentina, Chile and Uruguay, 1890-1940, Lincoln and London, University of Nebraska Press.

Mera Juan León, 1988 [1880], La escuela doméstica, in Pensamiento pedagógico ecuatoriano, Quito, Banco Central del Ecuador-Corporación Editora Nacional, p. 319-346.

MinistÈRe De L'ÉDuCATION, 1951, Libro del cincuentenario de la Fundación de colegios normales (Compilación de discursos), Quito, Imprenta del Ministerio de Educación.

Ministère De l'Instruction publique, 1900, Informe del Ministro de Instrucción Pública, Doctor José Peralta, Quito, Imprenta del Gobierno. 
—, 1907, Informe del Ministerio de Instrucción Pública al Congreso ordinario de 1907, Quito, Imprenta Nacional.

—, 1928, Informe del Ministro de Instrucción Pública Correos, Telégrafos, etc., Quito, Imprenta Nacional.

MORANT Isabel (dir.), 2006a, Historia de las mujeres en España y en América Latina, vol. III : Del siglo XIX a los umbrales del siglo XX, Madrid, Cátedra.

—, 2006b, Historia de las mujeres en España y en América Latina, vol. IV : Del siglo XX a los umbrales del siglo XXI, Madrid, Cátedra.

Moscoso Martha, 1996a, "Imagen de la mujer y la familia a inicios del siglo XX », Procesos, Revista Ecuatoriana de Historia, 8, p. 67-82.

-,1996b, Y el amor no era todo... Mujeres, imágenes y conflictos, Quito, ABYAYALA/Cooperación de los Países Bajos.

NovoA A., 1900, Recopilación de Mensajes dirigidos por el Presidente Eloy Alfaro, Guayaquil, Imprenta Novoa.

—, 1904, Recopilación de Leyes del Ecuador. Relaciones exteriores, Culto y Negocios eclesiásticos, Guayaquil, Imprenta del Telégrafo.

PÁEZ Alexei, 1986, El anarquismo en el Ecuador, Quito, Corporación Editora Nacional INFOC.

Penaherrera Padilla Blasco, 1991, El liberalismo en el Ecuador, Quito, Corporación Editora Nacional.

POTTAHST Barbara \& Eugenia SCARZANELla (dir.), 2001, Mujeres y naciones en América Latina. Problemas de inclusión y exclusión, Madrid / Frankfurt, Iberonamericana.

PRATT Mary Louise, 1993, "Las mujeres y el imaginario nacional en el siglo XIX », Revista de crítica literaria, 38, p. 51-62.

SINARDET Emmanuelle, 2005, «L'éducation féminine dans l'Équateur des années 1925-1945 », in Jean-Louis Guerena \& Mónica ZAPATA (dir.), Culture et éducation dans les Mondes Hispaniques - Essais en hommage à Eve-Marie Fell, Tours, Presses Universitaires François Rabelais, p. 265-277.

—, 2006, "José Peralta et l'école laïque: la révolution alfariste en question (18951906) », in Thomas GÓMEZ (dir.), La laïcité dans le monde ibérique, iberoaméricain et méditerranéen : idéologie, institutions et pratiques, Nanterre, Publidix, p. 295-309. 